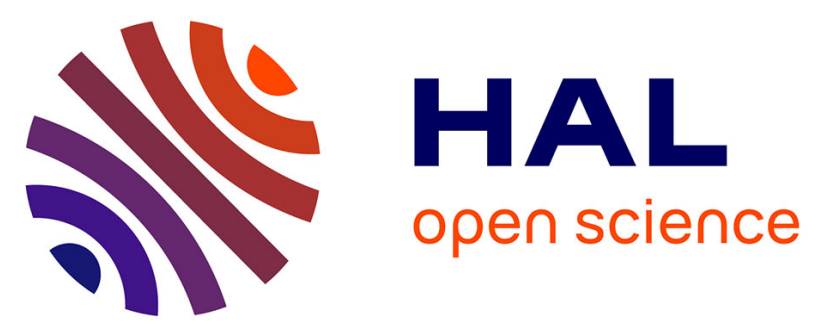

\title{
A multiscale mathematical model of avascular tumor growth to investigate the therapeutic benefit of anti-invasive agent
}

Benjamin Ribba, Olivier Saut, Didier Bresch, Emmanuel Grenier, Jean-Pierre Boissel

\section{To cite this version:}

Benjamin Ribba, Olivier Saut, Didier Bresch, Emmanuel Grenier, Jean-Pierre Boissel. A multiscale mathematical model of avascular tumor growth to investigate the therapeutic benefit of anti-invasive agent. Journal of Theoretical Biology, 2006, 243 (4), pp.532-541. 10.1016/j.jtbi.2006.07.013 . hal00385848

\section{HAL Id: hal-00385848 \\ https://hal.science/hal-00385848}

Submitted on 1 Dec 2020

HAL is a multi-disciplinary open access archive for the deposit and dissemination of scientific research documents, whether they are published or not. The documents may come from teaching and research institutions in France or abroad, or from public or private research centers.
L'archive ouverte pluridisciplinaire HAL, est destinée au dépôt et à la diffusion de documents scientifiques de niveau recherche, publiés ou non, émanant des établissements d'enseignement et de recherche français ou étrangers, des laboratoires publics ou privés.

\section{(c)(1)}

Distributed under a Creative Commons Attribution| 4.0 International License 


\title{
A multiscale mathematical model of avascular tumor growth to investigate the therapeutic benefit of anti-invasive agents
}

\author{
Benjamin Ribba ${ }^{a}$ Olivier Saut ${ }^{b}$ Thierry Colin ${ }^{b}$ Didier Bresch ${ }^{c}$ \\ Emmanuel Grenier ${ }^{d}$ and Jean-Pierre Boissel ${ }^{a}$
}

\begin{abstract}
${ }^{a}$ Institut de Médecine Théorique and Service de Pharmacologie Clinique, EA 3736, Faculté de Médecine R.T.H Laennec, Université Lyon 1, Rue Guillaume Paradin, 69376 Lyon Cedex 08, France

${ }^{\mathrm{b}}$ Mathématiques Appliquées de Bordeaux, CNRS UMR 5466 and INRIA futurs, Université Bordeaux 1, 351 cours de la libération, 33405 Talence Cedex, France

${ }^{\mathrm{c}}$ Laboratoire de Modélisation et de Calcul, CNRS UMR 6620, Université Joseph-Fourier, Rue des Mathématiques, 38051 Grenoble, France

${ }^{\mathrm{d}}$ Unité de Mathématiques Pures et Appliquées, CNRS UMR 5669, Ecole Normale Supérieure de Lyon, 69364 Lyon cedex 07, France
\end{abstract}

\begin{abstract}
With the aim to inhibit cancer growth and to reduce the risk of metastasis, pharmaceutical compagnies developed early 90's anti-metastatic agents called inhibitors of metalloproteinases. Despite the promising results obtained in preclinic, results of phase III trials have been somewhat disappointing for late stage cancer patients. With the aim to investigate mathematically this therapeutic failure, we developed a mechanistic-based model which integrates cell cycle regulation and macroscopic tumor dynamic. By simulating the model, we evaluated the efficacy of metalloproteinases inhibitors therapies. Simulation results predict the lack of efficacy of metalloproteinases inhibitors in advanced cancer patients. The theoretical model may help the design of prospective clinical trials to evaluate the efficacy of antimetastatic therapies.
\end{abstract}

Key words: Inhibitors of Metalloproteinases, Cytostatic agents, Mathematical modeling, Therapeutic development. 


\section{Introduction}

Mathematical models of cancer have been extensively developed with the aim to predict tumor growth and therapeutic strategies efficacy $(1 ; 2 ; 3 ; 4 ; 5)$. More complex multiscale models have been set up to address questions in the prediction of efficacy and toxicity of chemotherapy, radiotherapy $(6 ; 7)$, and to investigate the effect of cytostatic treatments such as anti-angiogenic and anti-maturation therapies (8).

In this paper, we're interested in cytostatic agents called the inhibitors of metalloproteinases (MMPi), developed to slow down cancer growth and to reduce the risk of metastasis. These agents have been designed to inhibit metalloproteinases (MMP), a group of enzymes which are known to play a major role in the degradation of basal membranes and extra-cellular matrixes and thus in cancer invasion. Despite the promising results obtained in animals models, the clinical development of these agents was rather disappointing. Retrospective analysis have highlighted a couple of potential reasons to explain this failure. Nonetheless, other studies are required to allow the resumption of further clinical trials.

The aim of this paper is to present a mathematical model which integrates basic understanding on the role of MMP in cancer growth in order to provide some explanation on the therapeutic failure and prospectively to help the design of further clinical trials of MMPi. Our model is composed by an agestructured cell cycle model with three populations. We applied Darcy's law to simulate spatially tumor dynamic. The efficacy of simulated MMPi treatment has been evaluated in term of tumor growth reduction.

\section{Biological background}

Solid cancers are characterized by an excessive proliferation of cells from epithelial layers. Due to several genetic mutations, cancer cells acquire the ability to produce growth signals and to loose sensitivity to anti-growth signals; the ability to escape from death processes, to replicate indefinitely, and to invade surrounding tissue to form metastasis (see (9) for further details).

During normal morphogenesis, cells evolve in a cell cycle which we often decomposed by a proliferative cycle where cells evolve to duplicate DNA and divide, and a quiescent phase where cells rest and wait for proliferative signals. It is known that proliferative cells can go to quiescence in case of anti-growth signals such as hypoxia, i.e. lack of oxygen, and/or nutrient deprivation. However, quiescent cells can turn back in the proliferative cycle if growth signals 
arise. In vivo tumor growth is often predicted by the Gompertz model $(1 ; 2)$ which features that at the beginning of the growth process, a large part of the cells are cycling in the proliferative phases which results in tumor exponential growth. As the number of cells increases, the local environmental can not supply cells with enough space, nutrient, and oxygen, and some cells are moving to quiescence. During cancer growth, new genetic mutations confer to the cells the ability to become unsensitive to anti-growth signals and thus to continue to proliferate.

Anti-neoplastic agents can be divided in two categories. The first, called cytotoxics, are aimed at killing cells by damaging their genetic contents. Standard chemotherapies and ionizing radiations belong to this class. Often their use is limited by the toxicity they generate on healthy tissue. Recently, the better understanding of the molecular circuits of cancer (10) encouraged the development of targeting treatments acting on molecular processes involved in tumor cancer in order to block cancer growth without damaging healthy cells. In interfering with the molecular pathways involved in cell proliferation (see (9) for a summary of these molecular pathways), these cytostatic agents have the ability to promote proliferative cells to move to quiescence and dormant cells to remain blocked in the quiescent phase of the cell cycle. Examples of such therapeutic agents are Cetuximab which prevent Epidermal Growth Factor (EGF) to bind EGFR and activate the mitogenic cascade (MAPK). The antiangiogenic agents Bevacizumab prevent the binding of the Vascular Endothelial Growth Factor (VEGF) to the receptors FLT-1 and FLK-1 and inhibit angiogenesis. In this paper, we are interested in anti-invasive cytostatic agents called the inhibitors of metalloproteinases (MMPi). Matrix Metalloproteinases (MMP) are a group of enzymes able to degrade the components of the Extra Cellular Matrix (ECM) (11). Many evidences in experimental studies prior to the 1990 reveal the role of MMP in promoting cancer growth, tissue invasion, and development of metastasis (12). Despite the structural differences between the members of the MMP family, non-specific inhibitors have been developed since at the time the precise role of each members was unknown and MMP action was seen globally to promote invasion and thus cancer growth (13). Preclinical studies have shown a significant benefit of MMPi to inhibit tumor growth and to prevent metastasis in animal models (14). Despite these encouraging results, clinical trials have been disappointing. Phase I showed toxicity which was not expected from preclinical studies. Results of Phase II were difficult to analyze (14). Indeed, the conventional endpoints used to measure efficacy of anti-cancer drugs, e.g., reduction of tumor volume, were not relevant for cytostatic agents since they do not kill cell but rather block proliferation. Phase III trials did not show any significant efficacy on the clinical endpoints $(14 ; 15)$.

Retrospective analysis provided few arguments to explain the failure of these therapeutic developments. It is admitted that a better understanding of each 
MMP is required before proceeding new development attempts.

\section{Methods}

We present a multiscale mathematical model of avascular tumor growth to investigate the role of MMPi treatment on cancer growth. The mathematical core is composed by an age-structured model where we distinguished between proliferative and quiescent cells. The cell cycle model is embedded in a macroscopic model based on Darcy's law to describe tumor spatial dynamic.

\subsection{Cell cycle model}

We developped an age-structured mathematical model of the cell cycle where cell cycle phase duration were set according to literature (16). We distinguished between the proliferative phases $P_{1}$ and $P_{2}$, and the quiescent phase $Q$. We modeled the 'Restriction point' $R$ (17) at the end of phase $P_{1}$ where environmental conditions are checked (11).

Cells evolve in time $t$ and in age $a$ in the proliferative phases of the cell cycle. We assumed that if the local number of cancer cells was above a threshold $T h_{t}$, then overpopulation was declared and leads cells at the end of $P_{1}$ to compartment $Q$ (quiescence) which is not age-structured. Quiescent cells can come back to the proliferative cycle (in phase $P_{2}$ ) as soon as the environmental conditions become appropriate again, i.e. the local number of cells goes below $T h_{t}$. The age-structured model can be written as follows:

$$
\left\{\begin{array}{l}
\frac{\partial P_{1}}{\partial t}+\frac{\partial P_{1}}{\partial a}=0 \\
\frac{\partial P_{2}}{\partial t}+\frac{\partial P_{2}}{\partial a}=0 \\
\frac{\partial Q}{d t}=(1-f(N)) P_{1}\left(a=a_{\max , P_{1}}\right)-\left[f^{\prime}(N)\right]^{+} Q
\end{array}\right.
$$

with the following initial conditions:

$$
\left\{\begin{array}{l}
P_{1}(a=0)=2 P_{2}\left(a=a_{\max , P_{2}}\right), \\
P_{2}(a=0)=f(N) P_{1}\left(a=a_{\max , P_{1}}\right)+\left[f^{\prime}(N)\right]^{+} Q
\end{array}\right.
$$

where $N$ denotes the local number of cells and $f(N)$ is a boolean function 


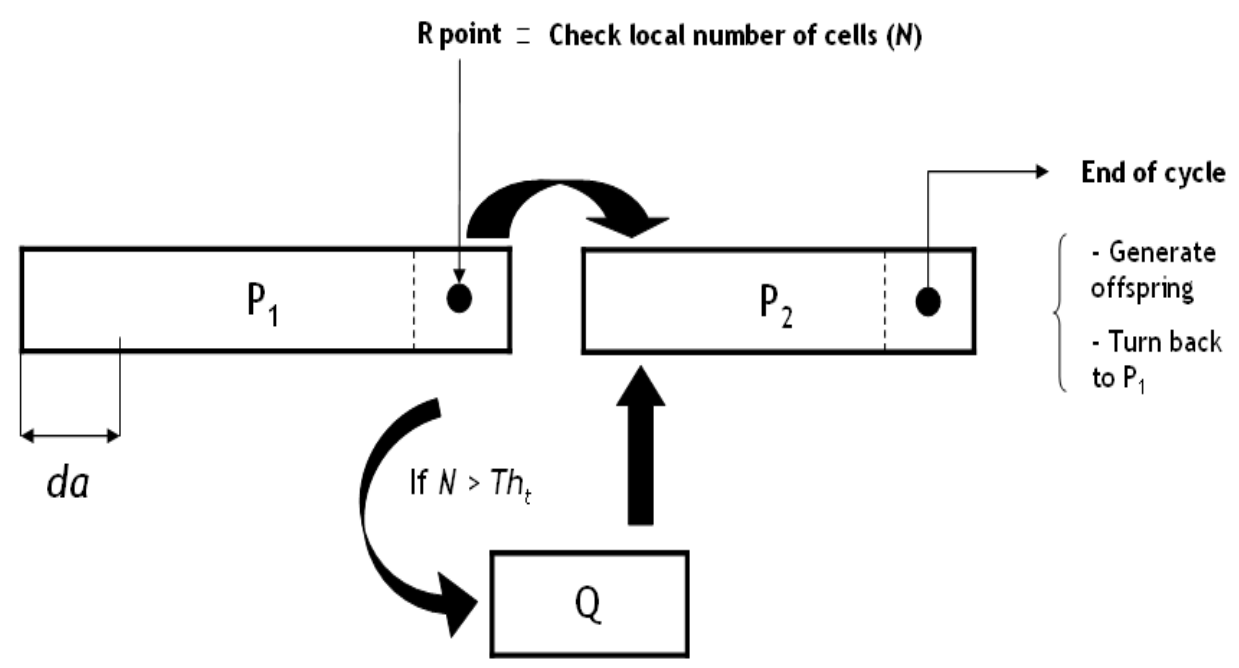

Fig. 1. The proliferative cycle is composed by two phases: $P_{1}$ and $P_{2}$. At the end of $P_{2}$, cells divide and new born cells begin their cycle in $P_{1}$. At the last age of phase $P_{1}$, we modeled the restriction point $R$, where external conditions are checked. If overpopulation occurs, proliferative cells go to the $Q$ compartment (quiescence). These cells can go back in the proliferative cycle (at the first step of phase $P_{2}$ ) if the external conditions allow it.

such that:

$$
f(N)=\left\{\begin{array}{l}
1 \text { if } N<T h_{t} \\
0 \text { otherwise }
\end{array}\right.
$$

See Figure 1 for a schematic representation of the cell cycle model.

\subsection{Macroscopic model}

We work in the framework of continuous meachnaics and we descrive the medium by using a velocity $v$ and a pressure $p$ that are related to the Darcy's law $(18 ; 19 ; 20)$ :

$$
v=-k \nabla p
$$

where $k$ denotes the media permeability. We refer to (18) for a precise description and justification of such models in the context of potential flows. Here, both quantities $v$ and $p$ are related to a mixture involving several species. These species are described by $n_{\varphi}(x, y, a, t)$ that represents the cell numbers (by volume unit) of cancer cells with spatial position $(x, y)$ at time $t$ with 
age $a$ in a given proliferative cycle phase $\varphi$. Applying the principle of mass balance, the advection equations are:

$$
\left\{\begin{array}{l}
\frac{\partial n_{\varphi}}{\partial t}+\frac{\partial n_{\varphi}}{\partial a}+\nabla \cdot\left(\mathbf{v} n_{\varphi}\right)=0, \quad \forall \varphi \in\left\{P_{1}, P_{2}\right\} \\
\frac{d n_{Q}}{d t}+\nabla \cdot\left(\mathbf{v} n_{Q}\right)=(1-f(N)) P_{1}\left(a=a_{\max , P_{1}}\right)-\left[f^{\prime}(N)\right]^{+} n_{Q}
\end{array}\right.
$$

where $N(x, y, t)=n_{Q}(x, y, t)+\sum_{\varphi} \int_{a} n_{\varphi}(x, y) \quad \forall \varphi \in\left\{P_{1}, P_{2}\right\}$ is the total number of cancer cells (by volume unit). Note that the quiescent phase $Q$ is not age-structured.

We denote $M$ the density of healthy tissue which evolves as follows:

$$
\frac{d M}{d t}+\nabla \cdot(\mathbf{v} M)=0
$$

and assume that $N(x, y, t)+M(x, y, t)=$ Constant $\quad \forall(x, y, t)$.

Integrating on ages $a$ equations 5 , adding them and using the relation $N+M$ is constant, one can find the following relation:

$$
\left[n_{\varphi}\right]_{a=0}^{a=a_{\max , \varphi}}+\nabla \cdot v=(1-f(N)) P_{1}\left(a=a_{\max , P_{1}}\right)-\left[f^{\prime}(N)\right]^{+} n_{Q} .
$$

Now using the initial conditions 2, we find the following equation for the velocity:

$$
\nabla \cdot v=P_{2}\left(a=a_{\max , P_{2}}\right)
$$

Thus, the pressure field $p$ satisfies the elliptic equation:

$$
-\nabla \cdot(k \nabla p)=P_{2}\left(a=a_{\max , P_{2}}\right) .
$$

We set $n_{\varphi}$ and $n_{Q}$ on part of the boundary where $v \cdot \nu<0$, with $\nu$ denoting the outgoing normal to the boundary . For the pressure $p$, we set $p=0$ on the boundary of the computational domain.

\subsection{MMP, membrane degradation and action of therapeutic}

It has been basically assumed that MMP are secreted by cancer cells and that the degradation of the matrix components was function of MMP. We modeled 
this as follows:

$$
\left\{\begin{array}{l}
M M P=\kappa_{M M P} N(t) \\
\frac{d E C M}{d t}=-\beta(M M P(t))
\end{array}\right.
$$

where $E C M$ stands for the basal membrane to be degraded by cancer cells and $\beta$ is an hyperbole-like function written as an 'Emax model':

$$
\beta(M M P(t))=\frac{\beta_{\max } \cdot M M P(t)}{\kappa_{\beta}+M M P(t)} .
$$

where $\beta_{\max }$ and $k_{\beta}$ are positive constants.

To simulate the evolution of a population of cancer cells under MMPi treatment we basically set $M M P(t)=0$ during the whole simulation which directly results in preventing ECM degradation and thus cancer cells dissemination in the surrounding tissue.

\subsection{Evaluation of the therapeutic benefit}

As the action of MMPi is aimed at promoting the passage of cells from proliferation to quiescence, we'll use the proportion of quiescent cells as the efficacy marker. In the following, $n_{Q}$ and $n_{Q}^{\star}$ will denote respectively the local number of quiescent cells without MMPi and with MMPi treatment. The absolute effect (AE) of the therapy will be compute as follows:

$$
A E=\frac{1}{T} \sum_{t=0}^{t=T}\left(\frac{\iint n_{Q}^{\star}(t) d x d y}{\iint N^{\star}(t) d x d y}-\frac{\iint n_{Q}(t) d x d y}{\iint N(t) d x d y}\right),
$$

where $N^{\star}$ is the total number of cells when MMPi treatment is applied, and $T$ the global simulation time. The evaluation of the therapeutic benefit will be done as follows:

$$
\left\{\begin{array}{l}
\text { if } A E>0 \text { the treatment is effective } \\
\text { otherwise the treatment is useless (or harmful) }
\end{array}\right.
$$

\subsection{Meaningful model parameters}

We identified two meaningful parameters which characterize both cell and tissue properties. At the cell level, the threshold $T h_{t}$ is used to decide wether cells 

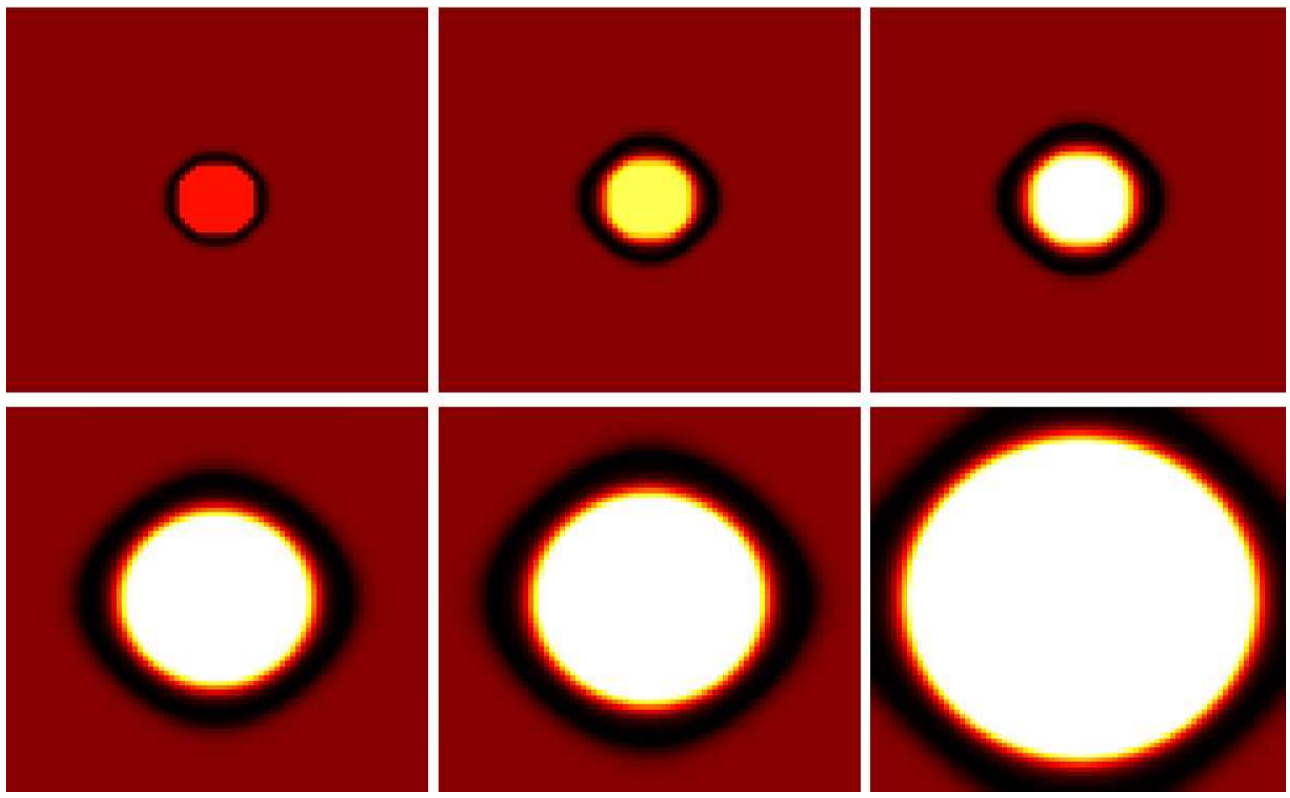

Fig. 2. From left to right and top to down. Illustration of ECM degradation and tumor cells in vitro invasion. Initially, tumor cells compose a central spot (red then light) surrounded by ECM (dark red). The MMP degrade the ECM (black ring) and allow tumor cells to invade the domain.

continue to proliferate or go to quiescence. Thus, an increase in this threshold characterizes a lack of sensitivity towards overpopulation. At the macroscopic level, the critical parameter is the tissue porosity noted $k$ in the Darcy's law (see Eq. 4). Different values of $k$ results in different cell spatial motion and in consequence different distribution in the cell cycle phases according to the regulation of the cell cycle (see Eq. 3).

\subsection{Computation domain and initial conditions}

We assumed that the domain is composed by healthy and cancer cells delimited from surrounding tissue by a membrane. Figures 2, 3, 4 present some illustrations of spatial-temporal evolution of cancer cells in lifelike geometries.

In the following we will keep the spatial configuration presented Figure 4 and note $k_{0}$ and $k_{1}$ the porosity constants of the healthy tissue and the membrane respectively with $k_{0}>k_{1}$ (see Figure 5). Initially, cells are distributed uniformly in the phases of the cell cycle.

Parameters used in the simulations are presented Table 1. 

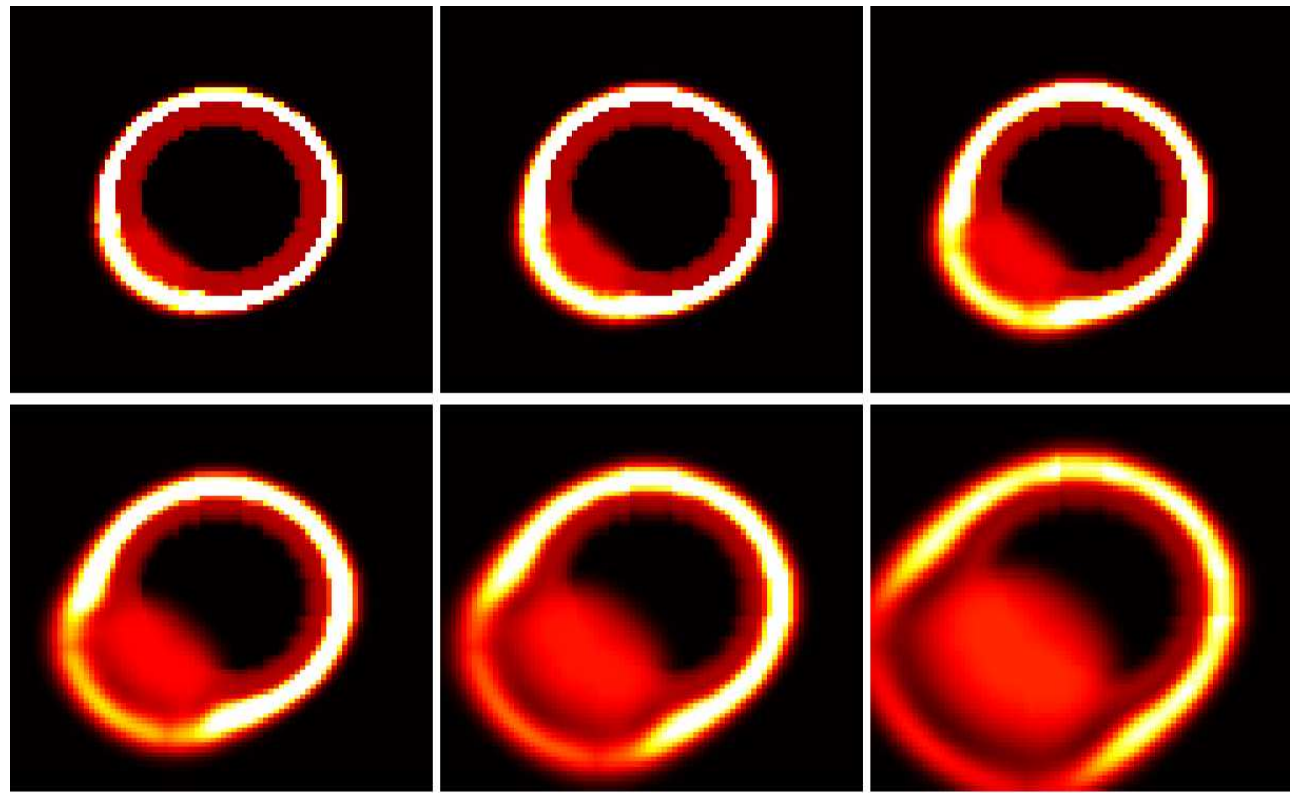

Fig. 3. From left to right and top to down. Illustration of in situ ductal carcinoma growth without basal membrane degradation. Initially, tumor cells compose a small area located at the bottom-left area of the epithelial layer. Tumor cells proliferate and deform the basal membrane (white ring) of the duct.
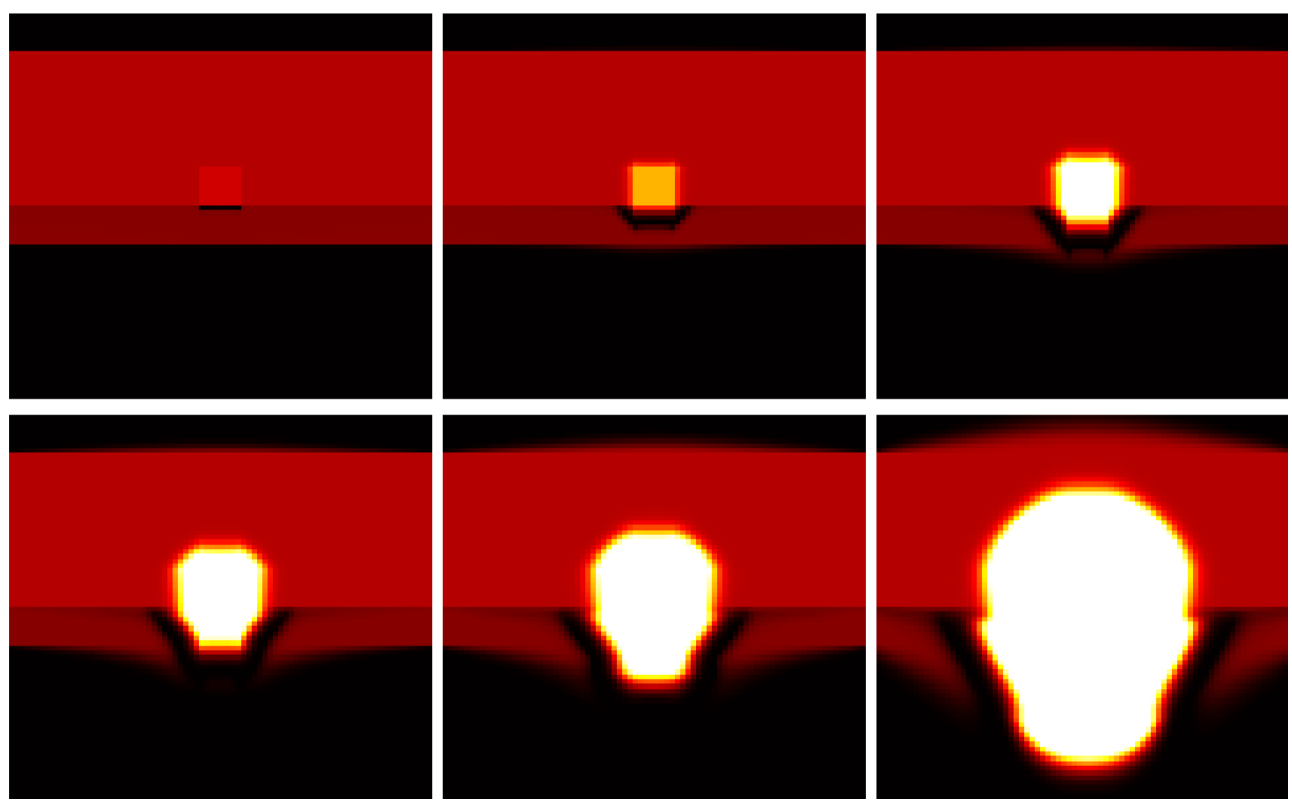

Fig. 4. From left to right and top to down. Illustration of invading epithelial cancer cells with basal membrane and ECM degradation. Initially, tumor cells compose a central small area adjacent to the membrane. Tumor cells proliferate, MMP degrade the membrane and cells invade the surrounding tissue. 


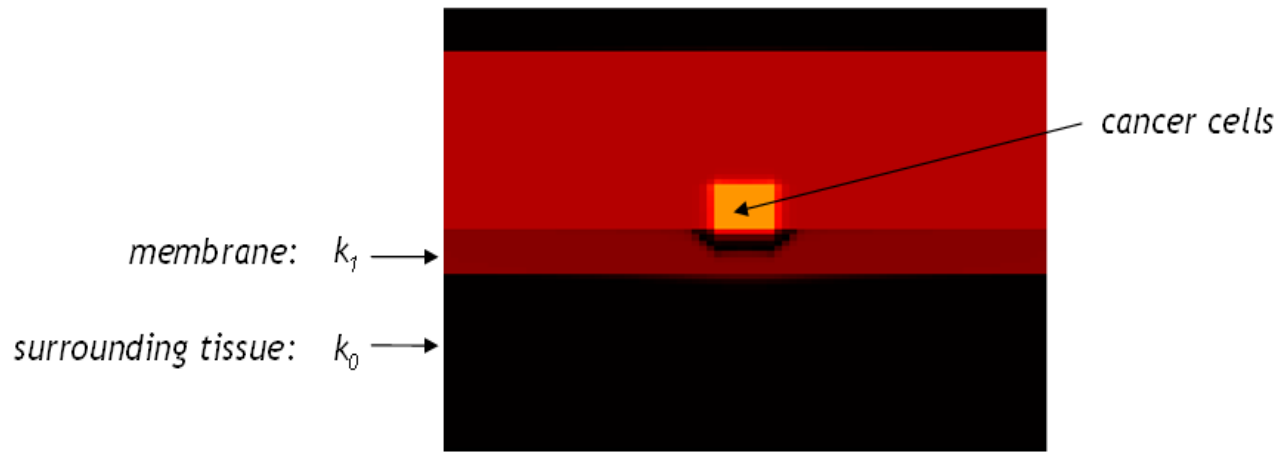

Fig. 5. Configuration used for the computer simulations aimed at evaluating the therapeutic benefit of MMPi treatment. Cancer cells, which form initially a small square, are embedded in the healthy tissue with porosity $k_{0}$ and placed against a basal membrane with higher porosity $k_{1}$.

Table 1

\begin{tabular}{|c|c|c|c|c|}
\hline Parameter & Description & Equation & Value & Source \\
\hline \hline$T$ & Global simulation time & & & \\
\hline$a_{\max , P_{1}}$ & Duration of $P_{1}$ & 1 and 2 & 20 & $(16)$ \\
\hline$a_{\max , P_{2}}$ & Duration of $P_{2}$ & 2 & 18 & $(16)$ \\
\hline$\kappa_{M M P}$ & MMP secretion coefficient & 10 & $5 \cdot 10^{-4}$ & \\
\hline$\kappa_{\beta}$ & parameter of function $\beta$ & 11 & 0.01 & \\
\hline$\beta_{\max }$ & parameter of function $\beta$ & 11 & 0.1 & \\
\hline
\end{tabular}

Dimensionless parameter values used for the numerical simulations.

\section{Results}

According to the macroscopic model (Darcy's law), the media porosity regulates the tendency for cells to move towards new areas or to remain at their spatial position. When the MMPi treatment is applied, it prevents membrane degradation. The porosity of the membrane should not allow cells to invade new tissue areas. In consequence, proliferating cells should remain at their spatial location. After a certain number of cycles, we expect that the local number of cells goes over the overpopulation threshold $T h_{t}$ which promote proliferative cells to move to quiescence. This will result in a positive absolute effect (see Eq. 12). 


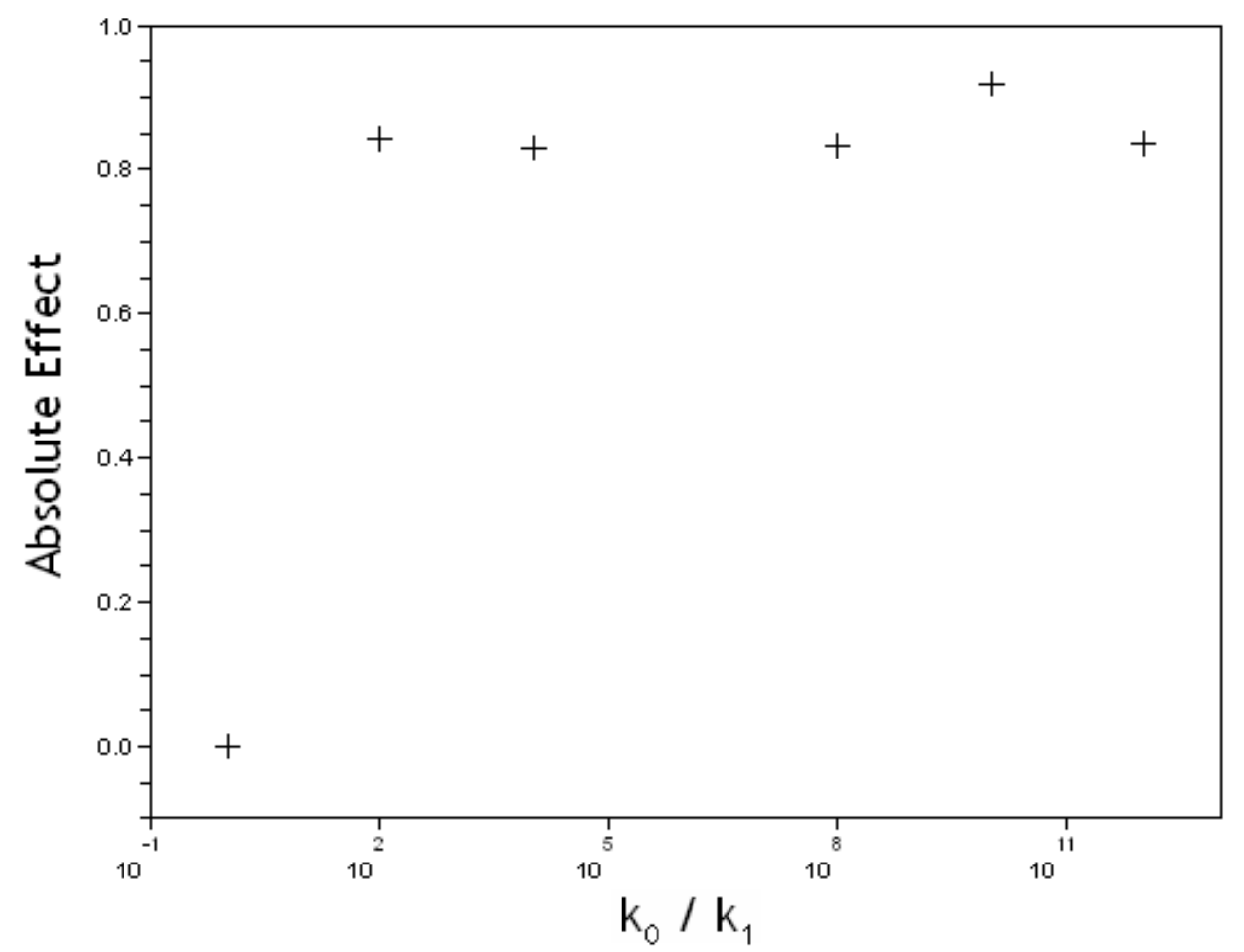

Fig. 6. Sensitivity of the absolute effect (AE) to the variation of the porosity ratio between surrounding tissue and basal membrane $\left(k_{0} / k_{1}\right)$.

\subsection{Influence of porosity ratio $k_{0} / k_{1}$ on the efficacy of MMPi treatment}

We first analyze how the efficacy of MMPi treatment is sensitive to the variation of the porosity ratio $k_{0} / k_{1}$. We ran six simulations for ratio porosity values $1,10^{2}, 10^{4}, 10^{8}, 10^{10}, 10^{12}$. For each porosity ratio values, we ran the simulations over 300 time steps, with and without MMPi treatment, and compute the absolute benefit with Eq. 12. Results are presented Figure 6.

While the ratio $k_{0} / k_{1}$ increases (the porosity of the membrane decreases respect the porosity of the surrounding tissue), we get as expected a positive absolute effect which indicates that the MMPi treatment is effective, i.e., it promotes proliferative cells to move massively to quiescence. Note that when the porosity of the surrounding tissue is equal to the membrane porosity, there's no therapeutic benefit to apply the MMPi treatment. Indeed, when the tissue porosity is equal to the membrane's one $\left(k_{0} / k_{1}=1\right)$, degradation does not have any effect on spatial availability for cells and thus repartition in phase $P$ and $Q$ of the cell cycle is not affected. 


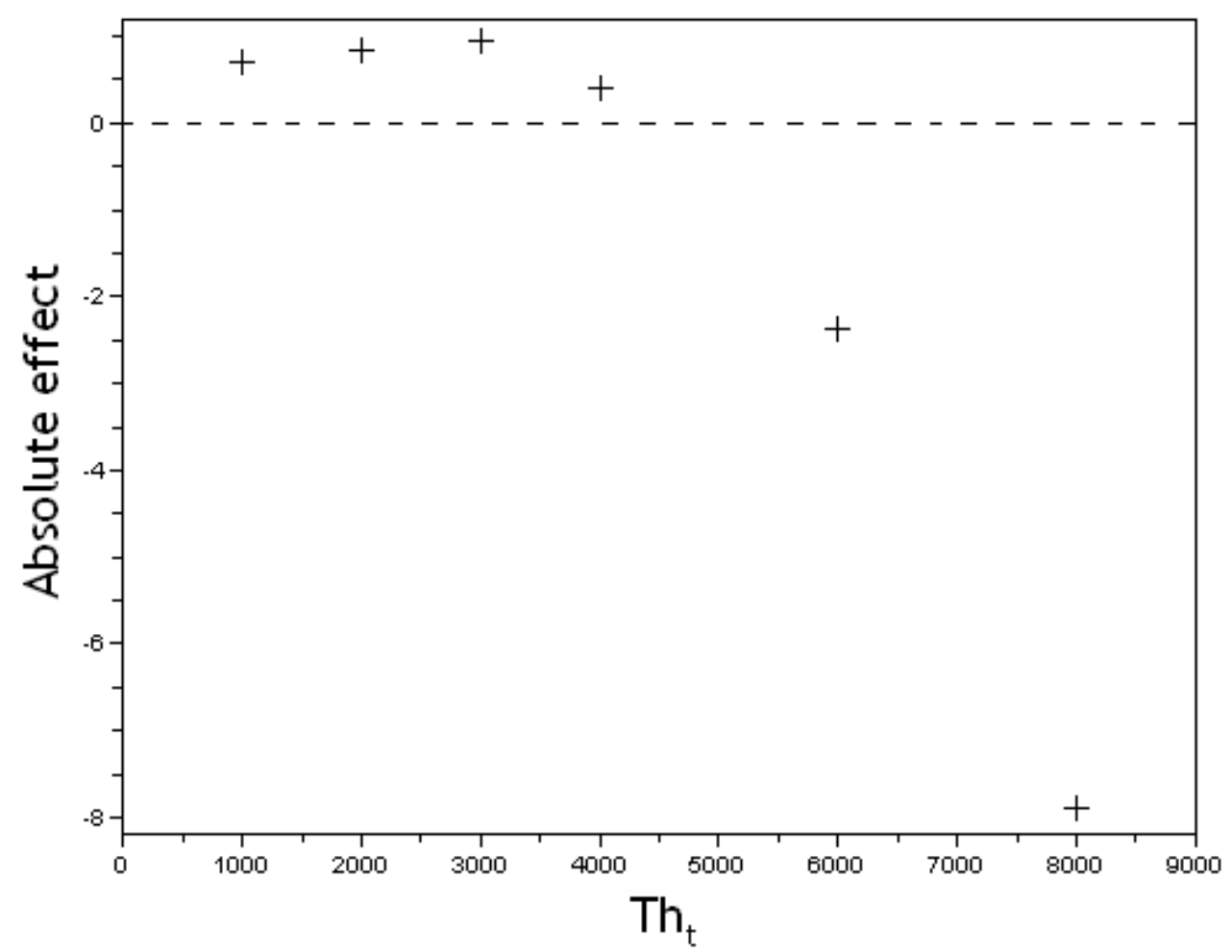

Fig. 7. Sensitivity of the absolute effect (AE) to variation of the overpopulation signal $\left(T h_{t}\right)$.

\subsection{Influence of overpopulation threshold $\left(T h_{t}\right)$ on the efficacy of $M M P i$ treatment}

We ran six simulations for overpopulation threshold values 1000, 2000, 3000, 4000, 6000, and 8000. For each of these values, we ran the simulations over 300 time steps, with and without MMPi treatment, and compute the absolute benefit with Eq. 12. For relatively low value of $T h_{t}$ we get a positive absolute effect which means that MMPi treatment is effective in slowing down tumor growth. When $T h_{t}$ is increased beyond a critical value (around 4000), the absolute effect collapses and becomes negative. Results are displayed Figure 7

Variation of the overpopulation threshold can change drastically the effect of the MMPi treatment: from beneficial to harmful.

We plotted Figure 8 the proportion of quiescent cells over time with and without MMPi for three different overpopulaton threshold values $(1000,4000$, and $8000)$. For the two first values of the threshold (1000, and 4000) the absolute benefit is positive (see Figure 7) while it is negative for the ultimate value (8000). Indeed, for $T h_{t}=8000$, quiescent cells in the case of MMPi treatment appear with a delay respect the situation without MMPi. The negative absolute effect results essentially from this delay. 


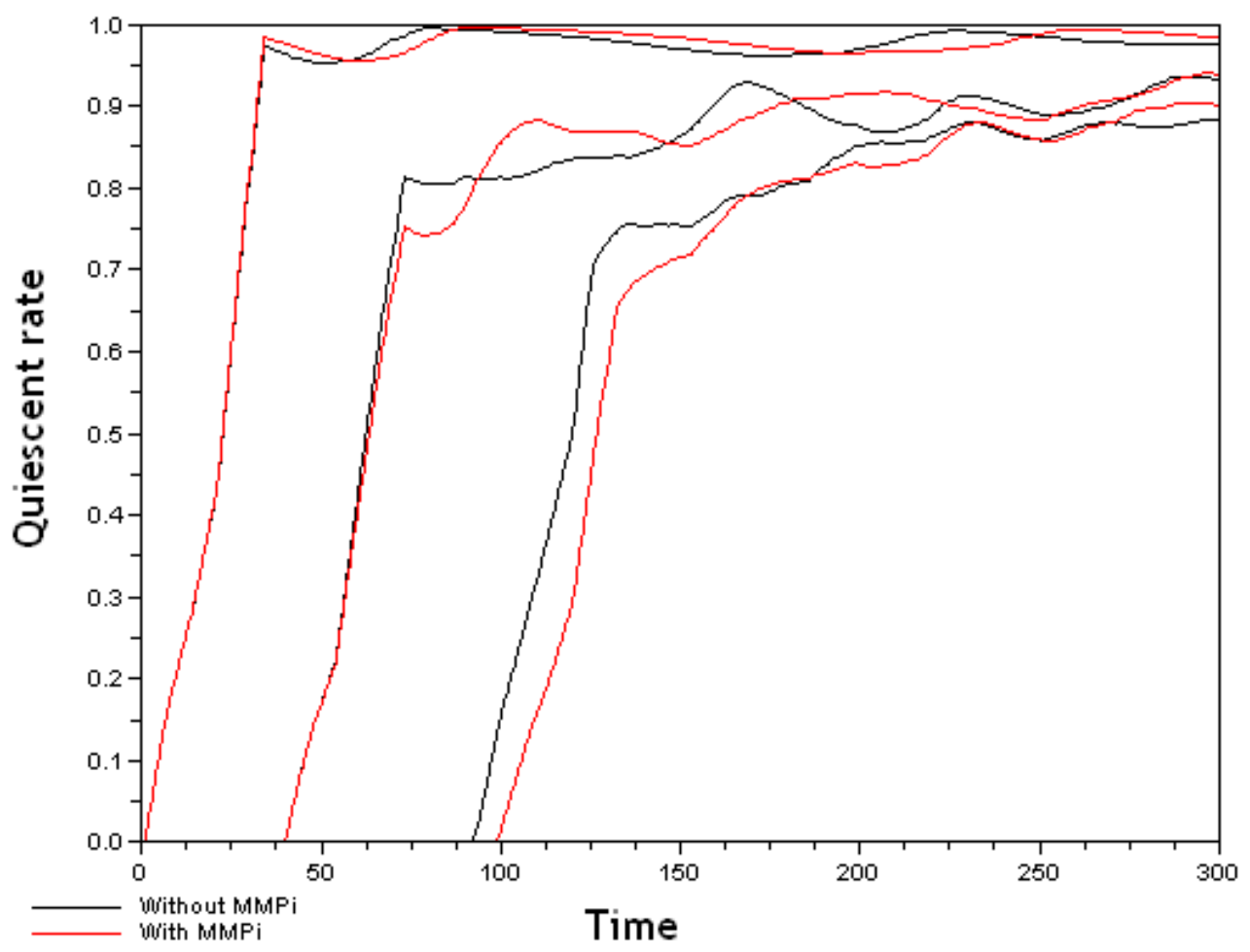

Fig. 8. Proportion of quiescent cells (quiescent rate) over simulation time (in hour) without MMPi (black curves) and with MMPi (red curves) for three overpopulation threshold values: $T h_{t}=1000,4000,8000$.

We show in Figure 9 the spatial configuration of quiescent and proliferative cells without MMPi (top) and with MMPi (bottom) from three different time (from left to right). The difference in the evolution of cell spatial configuration with and without MMPi can explain the delay observed in the appearance of quiescent cells when no MMPi is applied. When simulated the tumor growth with the presence of the treatment (bottom), tumor cells tend to extend uniformly within the computational domain which generate a homogeneous spheroid-like shape. On the contrary, in the absence of treatment, membrane degradation occurs and cells have tendency to be engulfed in the area when the membrane has been degraded. Cells at the center and the top of the initial tumor mass will be attracted towards the hole in the membrane and will not expand uniformly has before. In this case, the central part of the tumor mass will become quiescent more quickly than in the case when membrane degradation is prevented. 

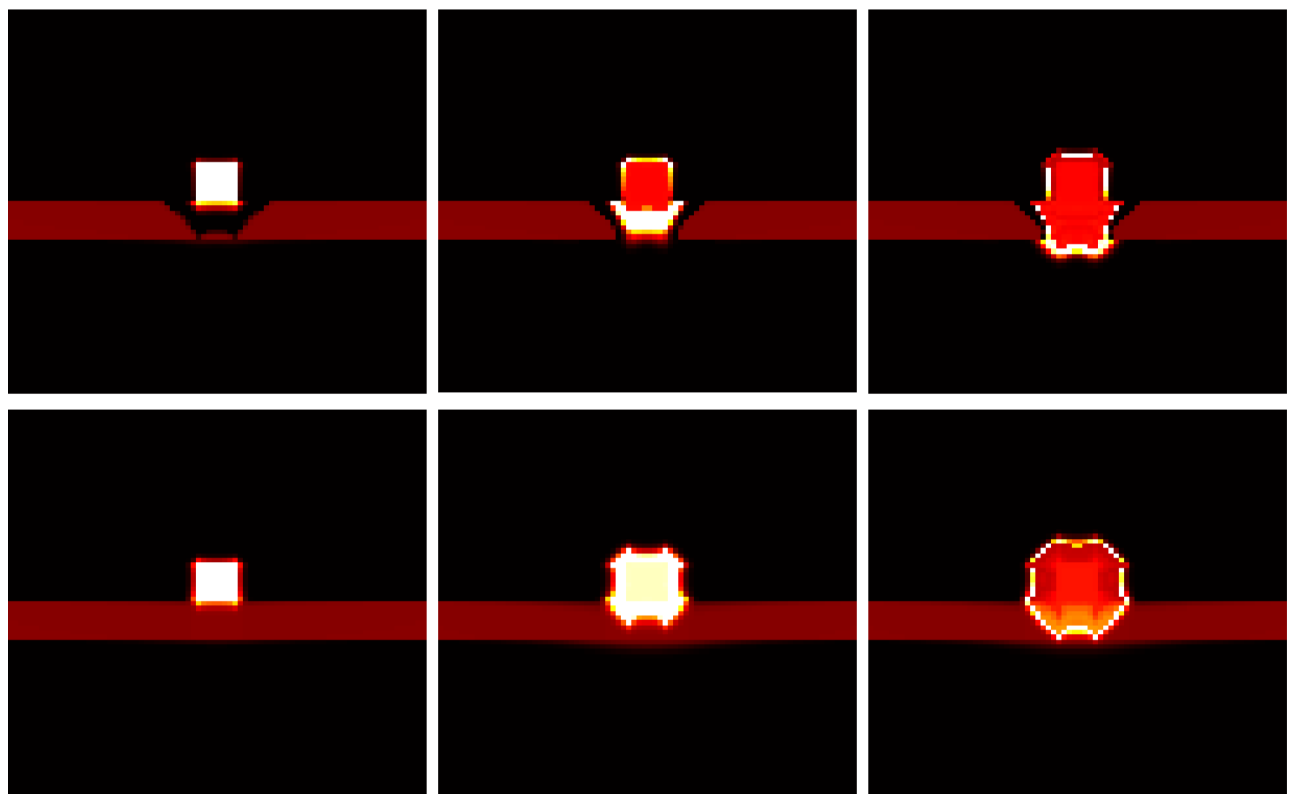

Fig. 9. From left to right: Spatial configuration of proliferative cells (white), and quiescent cells (red, composing the inner part of the mass) at time $t=40,128$, and 300. Top: Without MMPi. Bottom: With MMPi treatment. At time $t=128$, quiescent cells have appeared in the case of no MMPi treatment while at the same time, all cells are proliferative in the case of MMPi treatment.

\section{Conclusions}

MMPi were thought as promising therapeutic agents to slow down cancer growth and reduce the risk of metastasis. Their clinical development have failed despite good results obtained in preclinical trials. Retrospective analysis have highlighted the lack of knowledge on the role of MMP in cancer progression and several studies have emphasized the need to apply MMPi treatments on early stage cancer patients. It is known nowadays that MMP can also, according to the family enzymes they belong to and/or according to the patient disease stage, inhibit cancer growth (21). In this paper, we somehow investigated wether these conclusions could have been anticipated by integrating within a multiscale mathematical model the knowledge that was available at the time.

We built a mathematical model which integrates the basic actions of MMP within a macroscopic tumor growth regulated by a continuous cell cycle model. Simulation results obtained show that the overall efficacy of MMPi treatment may depend on the multiscale coupling between cell cycle regulation and tumor macroscopic behavior. Indeed, with the simple spatial configuration used, we showed that as cancer cells become insensitive to anti-growth signal, MMPi treatment efficacy decreases. 
However, as shown, the results obtained are sensitive to the spatial configuration of tumor cells and other initial configuration taken may lead to different and perhaps opposite results.

Nevertheless, the presented results are comparable to the recent conclusions raised by the retrospective analysis. Indeed advanced cancer are usually characterized by several mutations which give to cells a proliferating advantage by loosing their sensitivity to anti-growth signals. The simulation results emphasize the role of cells loosing sensitivity to anti-growth signals in the lack of efficacy of MMPi treatment. This may explain the lack of efficacy obtained during clinical development where MMPi treatment was given only at advanced stage cancer patients. However, the MMPi action preventing membrane degradation and tumor cells to invade the surrounding tissue should have been taken into account, together with the effect on cell proliferation, for a proper evaluation of therapy efficacy.

On a technical point of view, a three-dimensional tumor growth model could reveal new factors in the dynamics. We chose a continuous approach which provides density cells rather than actual cell number and we didn't consider cell shape, which has been shown to be important in the description of growth control process (22). To be more realistic, the present model in its $3 \mathrm{D}$ version should be coupled with an angiogenic model to allow simulation of MMPi treatment action on vascular tumor growth.

In conclusion, these results should be taken as theoretical and qualitative. Nonetheless, they highlight the need to consider multiscale regulation of tumor growth in the prediction of cancer treatment efficacy. Beyond the complexity of the molecular machinery, prediction of cytostatic therapies efficacy must integrate multiscale regulation of tumor growth via the cell cycle dynamic.

Nowadays, efforts are made to develop specific MMPi inhibitors to target the MMP which are effectively responsible for membrane degradation. Hopefully, a mathematical model as the one presented here may be used to optimize the design of further clinical development of these agents.

\section{Acknowledgements}

BR is founded by the ETOILE project: 'Espace de Traitement Oncologique par Ions Légers dans le cadre Européen'. We thank Mariama Diallo for reviewing litterature. 
[1] Brunton GF, Wheldon TE: The Gompertz equation and the construction of tumor growth curves. Cell Tissue Kinet 1980, 13:455460 .

[2] Bassukas ID: Comparative Gompertzian analysis of alterations of tumor growth patterns. Cancer Res 1994, 54:4385-4392.

[3] Skehan P, Friedman SJ: Deceleratory growth by a rat glial tumor line in culture. Cancer Res 1982, 42:1636-40.

[4] Hart D, Shochat E, Agur Z: The growth law of primary breast cancer as inferred from mammography screening trials data. $\mathrm{Br}$ J Cancer 1998, 78:382-387.

[5] Sachs RK, Hlatky LR, Hahnfeldt P: Simple ODE models of tumor growth and anti-angiogenic or radiation treatment. Math Comput Model 2001, 33:1297-1305.

[6] Ribba B, Marron K, Agur Z, Alarcon T, Maini PK: A mathematical model of Doxorubicin treatment efficacy for non-Hodgkin's lymphoma: investigation of the current protocol through theoretical modelling results. Bull Math Biol 2005, 67:79-99.

[7] Ribba B, Colin T, Schnell S: A multiscale model of cancer, and its use in analyzing irradiation therapies. Theor Biol Med Model 2006, 3(7).

[8] Arakelyan L, Merbl Y, Agur Z: A computer algorithm describing the process of vessel formation and maturation, and its use for predicting the effects of anti-angiogenic and anti-maturation therapy on vascular tumor growth. Eur J Cancer 2005, 41:159-167.

[9] Hanahan D, Weinberg RA: The hallmarks of cancer. Cell 2000, 100:57-70.

[10] Hahn WC, Weinberg RA: Modelling the molecular circuitry of cancer. Nat Rev Cancer 2002, 2:331-341.

[11] Kufe DW, Pollock RE, Weichselbaum RR, Bast RC, Gansler TS, Holland JF: Cancer Medicine, 6th ed. Hamilton (Canada): BC Decker Inc 2003.

[12] Giavazzi R, Taraboletti G: Preclinical development of metalloproteasis inhibitors in cancer therapy. Crit Rev Oncol Hematol 2001, 37:53-60.

[13] Zucker S, Cao J, Chen WT: Critical appraisal of the use of matrix metalloproteinase inhibitors in cancer treatment. Oncogene 2000, 19.

[14] Coussens LM, Fingleton B, Matrisian LM: Matrix metalloproteinase inhibitors and cancer: trials and tribulations. Science 2002, 295:2387-92.

[15] Hoekstra R, Eskens FA, Verweij J: Matrix metalloproteinase inhibitors: current developments and future perspectives. Oncologist 2001, 6:415-27.

[16] Potten CS, Kellett M, Roberts SA, Rew DA, Wilson GD: Measure- 
ment of in vivo proliferation in human colorectal mucosa using bromodeoxyuridine. Gut 1992, 33:71-78.

[17] Blagosklonny MV, Pardee AB: The restriction point of the cell cycle. Cell Cycle 2000, 1:103-110.

[18] Ambrosi D, Preziosi L: On the closure of mass balance models for tumor growth. Math Models Method Appl Sci 2002, 12:737-754.

[19] Greenspan HP: Models for the Growth of a Solid Tumor by diffusion. Stud Appl Math 1972, LI,4:317-340.

[20] Greenspan HP: On the growth and stability of cell cultures and solid tumors. J Theor Biol 1976, 56:229-242.

[21] Pavlaki M, Zucker S: Matrix metalloproteinase inhibitors (MMPIs): the beginning of phase I or the termination of phase III clinical trials. Cancer Metastasis Rev 2003, 22:177-203.

[22] Folkman J, Moscona A: Role of cell shape in growth control. Nature 1978, 273:345-349. 